\title{
Mode Transitions in a Model Reaction-Diffusion System Driven by Domain Growth and Noise
}

\author{
Iain Barrass ${ }^{\mathrm{a}}$, Edmund J. Crampin ${ }^{\mathrm{a}, \mathrm{b}}$, Philip K. Maini ${ }^{\mathrm{a}, *}$ \\ ${ }^{a}$ Centre for Mathematical Biology, Mathematical Institute, University of Oxford, 24-29 \\ St Giles', Oxford OX1 3LB, UK \\ ${ }^{b}$ Bioengineering Institute and Department of Engineering Science, The University of \\ Auckland, New Zealand
}

Received: 16 June 2005 / Accepted: 3 February 2006 / Published online: 6 June 2006 (C) Society for Mathematical Biology 2006

\begin{abstract}
Pattern formation in many biological systems takes place during growth of the underlying domain. We study a specific example of a reaction-diffusion (Turing) model in which peak splitting, driven by domain growth, generates a sequence of patterns. We have previously shown that the pattern sequences which are presented when the domain growth rate is sufficiently rapid exhibit a modedoubling phenomenon. Such pattern sequences afford reliable selection of certain final patterns, thus addressing the robustness problem inherent of the Turing mechanism. At slower domain growth rates this regular mode doubling breaks down in the presence of small perturbations to the dynamics. In this paper we examine the breaking down of the mode doubling sequence and consider the implications of this behaviour in increasing the range of reliably selectable final patterns.
\end{abstract}

Keywords Turing model $\cdot$ Schnakenberg · Spatial pattern · Robustness · Mode-doubling failure $\cdot$ Mode selection

\section{Introduction}

One of the central issues in developmental biology is to understand how spatial patterns arise as an embryo develops from a single fertilised cell into an adult. In 1952, Alan Turing proposed that a system of interacting chemicals could be driven unstable by diffusion and evolve into a spatial pattern. This was an example of self-organisation giving rise to emergent behaviour. Since this paper, there has been a great deal of mathematical research on this model framework, and it has been applied to several systems in development (see, for example, Meinhardt,

Dedicated to the memory of Lee A. Segel, a friend, mentor and inspiration.

${ }^{*}$ Corresponding author.

E-mail address:maini@maths.ox.ac.uk (P. K. Maini). 
1995; Murray, 2003). In this era of integrative biology, Turing's model is one of the very first examples of how integrating simple processes can give rise to complex outcomes, in this case, the combination of stabilising processes yields an unstable system.

When Lee Segel moved into mathematical biology, one of his first interests was in the problem of pattern formation and in trying to make sense of the counterintuitive results of Turing's paper. In 1972, the paper by Segel and Jackson showed how one could understand Turing's result at a conceptual and intuitive level, with an illustrative application in ecology.

The Turing model assumed that cells did not move but simply responded to chemical cues by differentiating accordingly. However, in other systems it is wellknown that cells do move, and the paper by Keller and Segel (1970) was the first to show that within the amoeboid cells of the slime mold, pattern formation could arise as the result of a chemotactic instability. This paper is now a classic and has served as an inspiration for models in many areas of development, as well as in medicine (specifically in models for wound healing and for cancer).

One of the main problems with the Turing reaction-diffusion theory for morphogenesis is that the patterns it produces can be very sensitive to small perturbations (see, for example, Bard and Lauder, 1974) and thus the model seems inappropriate when applied to robust pattern formation in developmental biology. Dillon et al. (1994) showed that the judicial choice of boundary conditions could greatly increase the robustness of certain patterns by making other patterning modes inadmissible.

More recently, Crampin et al. (1999) showed that domain growth could select certain patterns in a robust manner. Specifically, they showed that an exponentially growing domain could produce a sequence of mode-doubling pattern transitions, but that this broke down if the growth rate was too high or too low. While they were able to present an explanation for the former behaviour, it was not clear why the latter behaviour occurred.

Pattern sequences featuring mode doubling via regular insertion or splitting of peaks are common in diverse biological systems. For example, pigmentation patterns in fish (Kondo and Asai, 1995), skeletal patterning in vertebrate limbs (Hayes et al., 1998) and heterocyst spacing in cyanobacterium Anabaena (Yoon and Golden, 1998) feature such sequences. Given that regular splitting and insertion of peaks is a recurring feature in pattern forming systems in nature it is essential to understand why mathematical models of such reaction-diffusion systems exhibit mode-doubling failure when the growth rate of the domain is too slow. The aim of this paper is to address this question in the case of the exponentially growing domain.

In Section 2 we present the model system that we will study and illustrate how the standard numerical technique of discretising in space leads to a solution sequence in which mode-doubling breaks down under exponential growth in the domain with low growth rate. We show that this does not happen if we use a spectral method to solve the equations. We deduce that it is the erroneous introduction of asymmetries from the numerical scheme that causes mode-doubling failure. In Section 3 we use the numerical bifurcation package AUTO (Doedel et al., 2001) to trace the bifurcation diagram for the fixed domain case with domain length as the 
bifurcation parameter. From this, we note that mode-doubling failure appears to occur when the solution on the growing domain has to trace an unstable portion of a solution branch. We examine this in more detail in Section 4 by considering the form of the unstable solution at the onset of instability.

Having seen that it is the noise in the numerical scheme that drives modedoubling breakdown, we then consider in Section 5 other forms of noise which may arise in a biological context, namely noise in the parameter values. In Section 6 we consider our results in light of the problem of robust pattern selection and present conclusions in Section 7.

\section{Pattern sequences on the growing domain}

As a concrete example we use the Schnakenberg kinetics, which arise from an application of the law of mass action to the trimolecular reaction scheme

$$
\begin{aligned}
& B \stackrel{k_{1}}{\rightleftharpoons} X, \\
& A \stackrel{k_{2}}{\rightarrow} Y, \\
& 2 X+Y \stackrel{k_{3}}{\longrightarrow} 3 X .
\end{aligned}
$$

We chose this model mechanism because of its relative simplicity and because it has been well-studied (Murray, 2003). In this case, the species $X$ acts as the activator.

The non-dimensionalised kinetics with $u(x, t)$ the concentration of $Y$ and $v(x, t)$ the concentration of $X$ on the one-dimensional domain of length $L(t)$ growing exponentially, but rescaled continuously onto the unit interval $x \in[0,1]$, are

$$
\begin{aligned}
\frac{\partial u}{\partial t} & =\frac{1}{L^{2}} \frac{\partial^{2} u}{\partial x^{2}}+a-u v^{2}-\rho u, \\
\frac{\partial v}{\partial t} & =\frac{d}{L^{2}} \frac{\partial^{2} v}{\partial x^{2}}+b+u v^{2}-v-\rho v, \\
\frac{\mathrm{d} L}{\mathrm{~d} t} & =\rho L,
\end{aligned}
$$

where $\rho$ is the domain growth rate (see Crampin et al., 1999 for details). The terms $-\rho u$ and $-\rho v$ describe the dilution effects due to the local expansion of the domain. In this paper we fix the kinetics parameters $a=0.9$ and $b=0.1$. We also take the ratio of diffusion coefficients $d=0.06$ so that the inhibitor diffuses more rapidly than the activator and Turing pattern expression is permitted on the static fixed-length domain. Patterns arising in this system are in the form of spike solutions (Iron et al., 2004), and the activator and inhibitor profiles are out of phase.

For the zero-flux boundary conditions to which we restrict our attention the corresponding uniform steady state on the static domain has $(u, v)=(0.9,1)$. Our initial conditions are small amplitude spatially uncorrelated random perturbations from this steady state and an initial domain length of $L(0)=1$. 
This system was studied in detail by Crampin (2000) for the case of exponential domain growth. Here, we solve the system numerically using the NAG library routine D03PCF which implements a finite difference method-the method of lines - with integration in time using a backward differentiation formula method. In this case we see the canonical mode-doubling sequence through peak splitting. In the mode-doubling sequence the number of peaks (the 'pattern mode') doubles each time the domain doubles in length. For this model, such a sequence occurs by splitting of each existing peak at regular intervals, while other models show mode doubling via peak insertion, where new peaks appear in between existing peaks as the length of the domain doubles (Crampin et al., 2002).

The first pattern to develop as the domain grows (the first admissible pattern) is a half peak which consists of a peak located on either the left- or the righthand boundary. Which of these two pattern polarities occurs depends on the initial conditions. However, after the transition from this first pattern mode we see that all subsequent pattern modes are of the same polarity (with internal peaks, rather than peaks located on the boundaries). This locking in of polarity is a consequence of the peak splitting mechanism (and a sequence of patterns of opposite polarity, with peaks located on the boundaries, is observed for peak insertion).

The mode-doubling sequence is characterised by smooth transitions between the quasi-steady patterns. That is, there is a separation of timescales between the dynamics of the reaction-diffusion system and the growing domain, the growth being on the slow timescale and the chemical dynamics on a fast timescale. However, when $\rho$ increases so that the separation of timescales becomes less apparent we see that there is no longer a recognisable sequence of quasi-steady patterns. Figure $1 \mathrm{~b}$ shows such a pattern sequence with $\rho=0.05$. The lack of applicability of the quasi-steady state assumption in such cases is clear and we can no longer assume that the processes of growth and pattern formation are decoupled.

At the other extreme of the domain growth rate $\rho$ we consider the case $\rho=$ 0.0001 . Numerical solutions of the system (4)-(6) obtained by discretising the system on a spatially uniform mesh exhibit the phenomenon of mode-doubling failure shown in Fig. 2. In this case the sequence of patterns is not the same

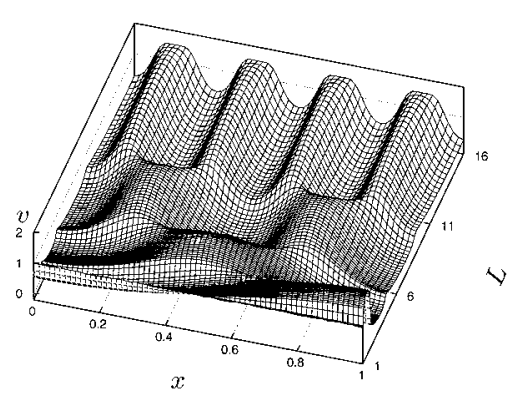

(a) $\rho=0.001$

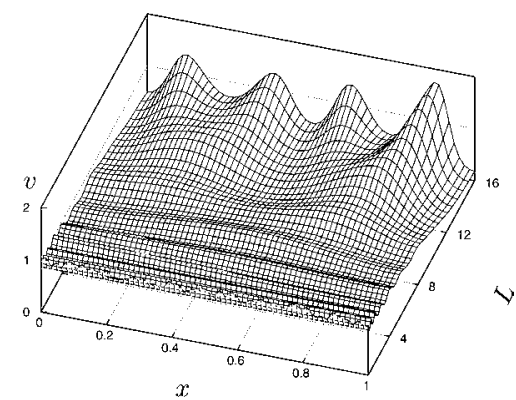

(b) $\rho=0.05$

Fig. 1 Activator concentration profile sequences on the growing domain with large growth rate. (a) Mode doubling evident in the quasi-steady pattern sequence with $\rho=0.001$. (b) $\rho=0.05$ and there is no quasi-steady evolution. 


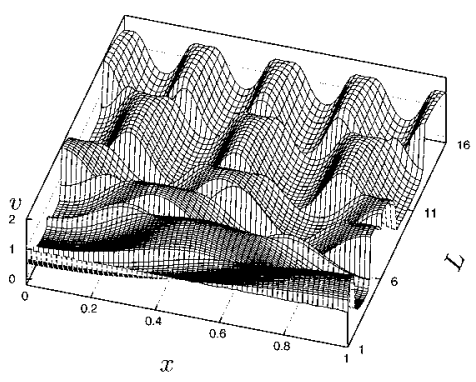

(a)
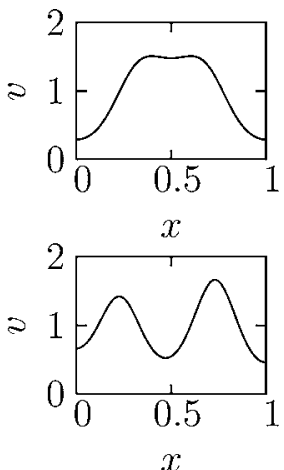

$x$
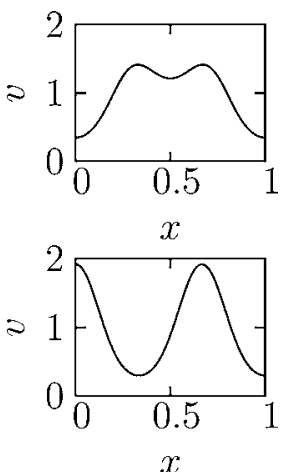

(b)

Fig. 2 Mode doubling breaks down at low domain growth rates when (4)-(6) is solved using a spatial discretisation explicit method. (a) A typical activator concentration profile for $\rho=0.0001$; (b) snapshots of the profile showing the asymmetric splitting of the single interior peak which forces the failure of mode doubling.

as before (compared to Fig. 1(a)) and there is not a uniform splitting of peaks leading to doubling of the pattern mode each time the domain length doubles. This phenomenon, whereby the mode-doubling sequence is not realised for very slow growth rates $\rho$, is what we refer to as mode doubling failure.

In this case we do see recognisable quasi-steady patterns, but the transitions between these are more abrupt. In part this is related to the difference in the timescales of domain growth and pattern formation, but we shall see later that there are further qualitative differences between the two cases of mode-doubling failure and mode-doubling success. Figure 2 illustrates the mechanism by which peak splitting fails to generate mode doubling, via asymmetric splitting of a peak. This asymmetric splitting of the single central peak, rather than the symmetric splitting and separation of peaks in Fig. 1(a) suggests that mode-doubling failure is related to the symmetry of the system and occurs when there is a sufficient degree of asymmetry introduced in this splitting process. We investigate this further below.

Due to the form of Eqs. (4)-(6), we expect mode-doubling to be the only form of behaviour, appealing to the invariance of the system under the mapping $x \mapsto 1-x$. The asymmetry that is necessary to lead to a breakdown of mode doubling must therefore be introduced by errors arising during numerical solution of the equations, or persist from the initial state. Should that be the case we would wish to understand the nature by which mode-doubling transition may be sensitive to these numerical issues. Before we examine this sensitivity, however, we note that, rather than a 'critical growth rate' for a given transition-below which mode doubling persistently fails, and above which it persistently succeedswe observe the presence of a critical growth rate window (Fig. 3). For growth rates within this window there is a given probability for successful mode doubling where mode doubling is deemed to have succeeded if the pattern expressed at domain length $L=5$ is close to the stable mode 4 pattern. For a given numer- 


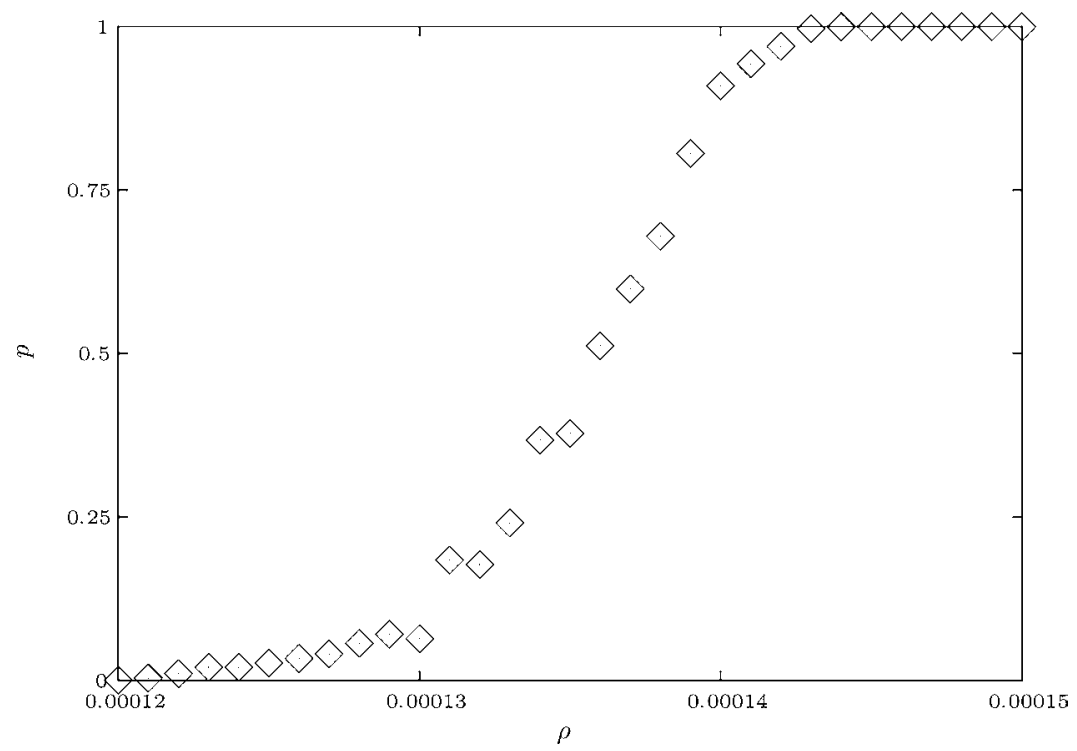

Fig. 3 Indicative critical growth rate window for the PDE system (4)-(6) as solved by an explicit finite difference scheme on a uniform spatial mesh. The mode-doubling probability for a given growth rate is estimated by sampling from 200 realisations with initial conditions (at domain length $L=1$ ) randomly selected small perturbations from the homogeneous steady state.

ical method, whether or not mode doubling occurs is determined by the initial state.

As an alternative to this basic numerical method we also consider a spectral method reduction of the PDE system (4)-(6) to a finite ODE system. A natural choice of basis for the system with zero-flux boundary conditions is the Fourier cosine series

$$
U(x, t)=\sum_{n=0}^{\infty} U_{n}(t) \cos (n \pi x) \quad \text { and } \quad V(x, t)=\sum_{n=0}^{\infty} V_{n}(t) \cos (n \pi x)
$$

Using these series expansions, we can derive from the system (4)-(6) an alternative ODE form which preserves the symmetry of the initial state. Without a direct translation of the initial conditions from the Schnakenberg PDE system to the finite ODE system we therefore, consider this ODE form as a distinct system.

The truncation to $N$ modes in each of the series $U$ and $V$, which represent the true solutions $u$ and $v$, respectively, of Eqs. (4)-(6), gives rise to the ODE system

$$
\begin{aligned}
& \frac{\mathrm{d} U_{0}}{\mathrm{~d} t}=a-c_{0}-\rho U_{0}, \\
& \frac{\mathrm{d} V_{0}}{\mathrm{~d} t}=b+c_{0}-(1+\rho) V_{0},
\end{aligned}
$$


and, for $1 \leq n \leq N-1$,

$$
\begin{aligned}
\frac{\mathrm{d} U_{n}}{\mathrm{~d} t} & =-c_{n}-\left(\frac{n^{2} \pi^{2}}{L^{2}}+\rho\right) U_{n}, \\
\frac{\mathrm{d} V_{n}}{\mathrm{~d} t} & =c_{n}-\left(\frac{\mathrm{d} n^{2} \pi^{2}}{L^{2}}+1+\rho\right) V_{n}, \\
\frac{\mathrm{d} L}{\mathrm{~d} t} & =\rho L,
\end{aligned}
$$

where $c_{i}$ represents the truncated Fourier cosine series reduction of the cubic term $u v^{2}$ in the Schnakenberg kinetics.

Solving this new system (8)-(12) numerically, again using a backward differentiation formula method for the time integration, we are able to reproduce the phenomenon of mode doubling by symmetric peak splitting. With the truncation to $N=40$, the steady state patterns expressed (with $\rho=0$ ) in the two systems (4)(6) and (8)-(12) are in close agreement. In addition, however, we find that, under this scheme, mode doubling appears to persist at arbitrarily small domain growth rates. This discrepancy reinforces the view that numerical inaccuracy, and the erroneous introduction of anti-symmetric Fourier modes, plays a significant rôle in mode-doubling failure.

\section{Bifurcation structure for the Schnakenberg kinetics}

We have established that the influence of numerical errors whilst solving the Schnakenberg system (4)-(6) with an explicit finite differences scheme with the method of lines may be sufficient to drive the system towards a mode-doubling failure state. With an alternative reduction to an ODE system we observe the persistence of mode doubling at arbitrarily small growth rates. In this section we examine in depth how the mode-doubling mechanism is susceptible to the influence of erroneous Fourier mode growth.

To this end, we consider the bifurcation structure of the Schnakenberg system on the static domain, with the domain length $L$ as the primary bifurcation parameter. We generate this structure, with the primary branches shown in Fig. 4, using the numerical bifurcation and continuation package AUTO taking as the solution measure the left-hand boundary value of the activator $V$. In Fig. 4, we also illustrate our labelling scheme for the primary branches which are expressed as large-amplitude patterns. The primary branches are labelled by a mode number which denotes position of the branch (in the increasing $L$ direction) on the bifurcation diagram and corresponds to the wavenumber of the branch in the linear system; a superscript + or - which denotes the polarity of the branch: branches labelled by + have left-hand boundary activator concentration greater than that of the uniform steady state; a subscript $u$ or $s$ which denotes instability or stability, respectively, of the branch region. Unstable branch regions in Fig. 4 are additionally indicated by broken lines, whereas stable regions have solid lines. 


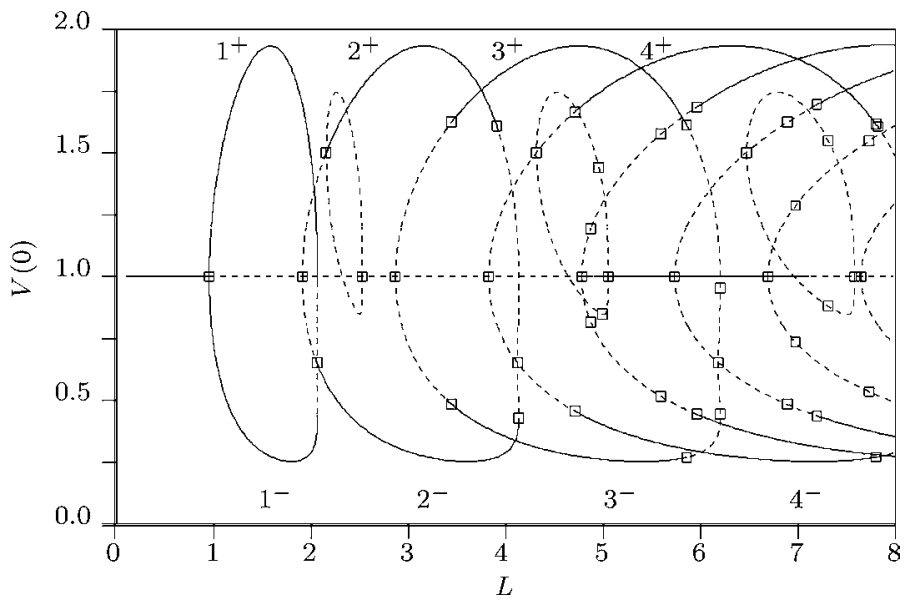

Fig. 4 The bifurcation structure of the system (8)-(11) with the domain length $L$ as primary bifurcation parameter and $\rho=0$ so that we ignore the dilution terms. For clarity we show only the primary branches; during quasi-steady evolution the secondary branches (which are unstable) are not traced.

With slow domain growth, Figs. 1(a) and 2(a) show evidence of quasi-steady behaviour in the activator concentration profile. Both of these pattern sequences exhibit the long-term expression of a pattern before the rapid re-organisation of this pattern to a further long-expressed pattern, adiabatically following primary bifurcation branches. We therefore consider how these quasi-steady state patterns, and their re-organisation, tie in to the steady states of the static domain.

By visual inspection we can see that the quasi-steady patterns expressed during domain growth are close to the steady-state patterns on the static domain. We note that the effects of the dilution terms $-\rho u$ and $-\rho v$, as well as the differences resulting from the different ODE reductions, are small as $\rho$ is small. Furthermore, we can project the evolving pattern onto the one-dimensional domain using the same solution measure as for the bifurcation structure. In Fig. 5 we see how these two profiles trace the steady-state branches for significant periods of time during domain growth. For this reason, then, we refer to the quasi-steady patterns that are expressed by the labels of the steady-state branches to which they are close.

We also see that mode-doubling failure appears to be related to the tracing of an unstable branch. In Fig. 5(a), we see that the region $4_{u}^{-}$is closely traced by the dynamic solution until the branch $4^{-}$becomes stable. In Fig. 5(b), where mode doubling fails, we see that this same branch region is incompletely traced before departure to the stable branch $3_{s}^{+}$.

In Section 4 we examine the nature of mode-doubling failure in depth and its relation to this tracing of an unstable branch region. Before then, however, we note that for the solutions of the system (8)-(12), where mode doubling persists for all slow growth rates, tracing of the unstable $4_{u}^{-}$is observed. 


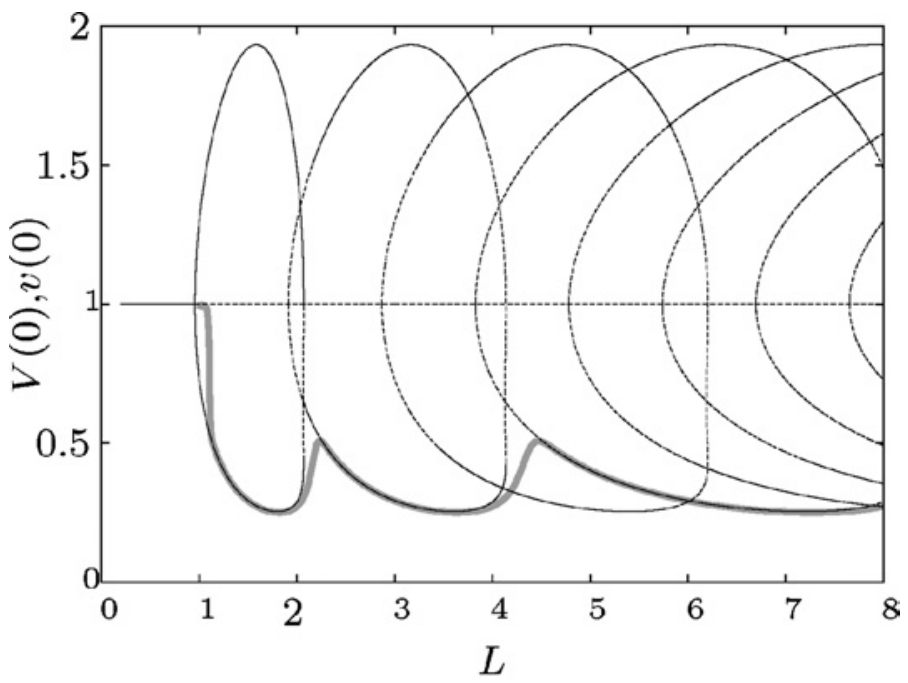

(a)

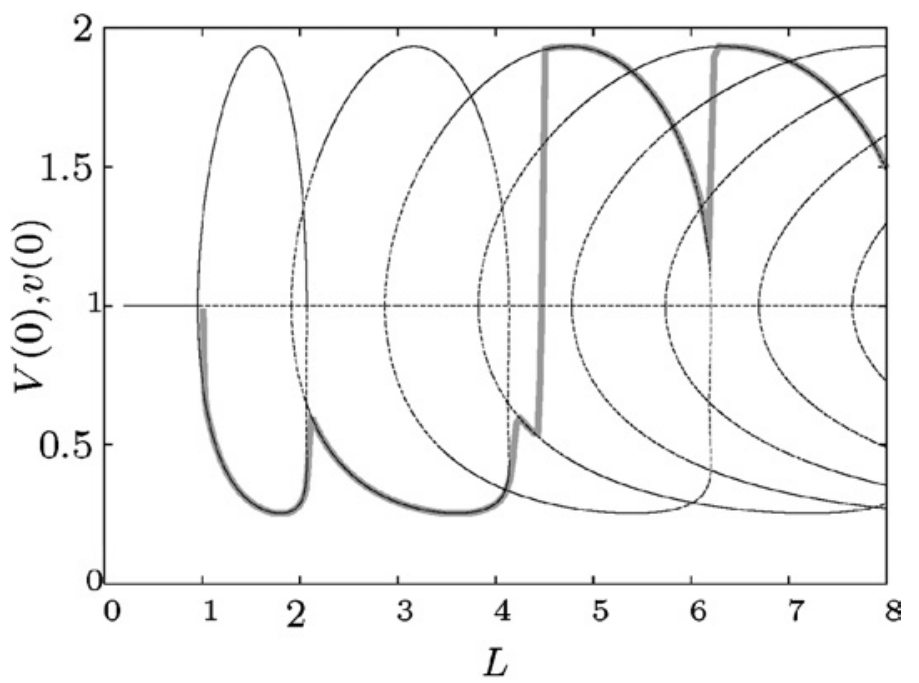

(b)

Fig. 5 Projection of the pattern sequences of Figs. 1(a) and 2(a)—grey trajectories—onto the bifurcation diagram of Fig. 4.

\section{Mechanism of mode-doubling failure}

The steady state of the system for the traced region $4_{u}^{-}$on the static domain is a saddle point with a one-dimensional unstable manifold. Failure of mode doubling is the departure of the phase point along the unstable manifold away from the 


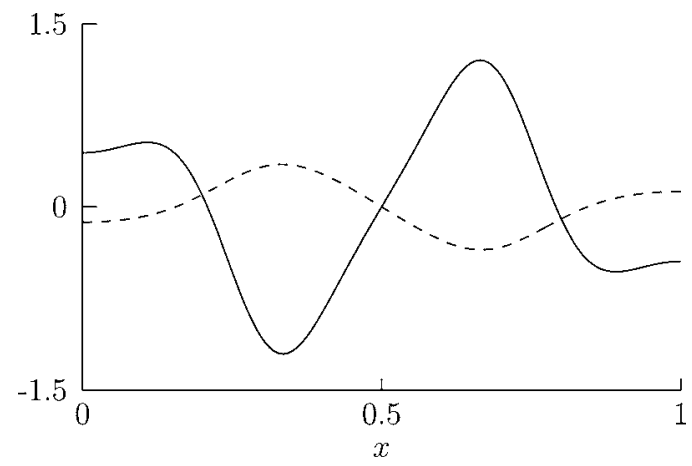

Fig. 6 The unstable eigenfunction on the branch $4_{u}^{-}$corresponding to the eigenvalue 0.0109 for domain length $L=4.62$. The profile of the eigenfunction does not change significantly with variation in $L$. The solid line details the $V$-component of the eigenfunction and the broken line the $U$-component.

branch $4_{u}^{-}$to either of the branches $3_{s}^{ \pm}$. This mechanism accounts for the occurrence of mode-doubling failure once the growth rate of the domain becomes too slow. At slower growth rates the time spent in the presence of the unstable manifold is increased. Consequently there is a tendency for greater movement along the unstable manifold. At some point, in the nonlinear regime, the phase point lies within the basin of attraction of either of the alternative stable branches. Certainly, mode doubling fails if the dynamic solution is not within the basin of attraction of the newly stable $4_{s}^{-}$(around $L \approx 4.71$ ).

Mode-doubling failure, then, is an expression of large deviation along an unstable manifold. The unstable eigenfunction along $4_{u}^{-}$(shown for the point $L=4.62$ in Fig. 6) is found to be anti-symmetric. The presence of this anti-symmetric unstable eigenfunction drives the expression of asymmetric peak splitting as the means of mode-doubling failure. Furthermore, the symmetry properties of the two branches $2^{-}$and $4^{-}$for Eqs. (4)-(6) and (8)-(12) explain why mode doubling is the natural behaviour of the dynamic system. Finally, the occurrence of mode-doubling failure in Eqs. (4)-(6) is indeed promoted by the possibility of erroneous Fourier mode introduction by the numerical method.

In addition to the existence of a critical growth rate for mode doubling in the system (4)-(6) as solved using a spatial discretisation method we are in a position to explain the appearance of the critical growth rate window of Fig. 3. Similarly to the robustness problem for Turing pattern formation on the static domain, there is sensitivity to the initial conditions for whether mode doubling succeeds or fails.

At some growth rates the success of mode doubling is sensitive to the initial state and the subsequent propagation of numerical error. The growth rate $\rho=0.000136$ corresponds to a mode-doubling probability close to 0.5 in the critical growth rate window of Fig. 3. Mode doubling and failure of mode doubling are illustrated in Fig. 7. These solutions have distinct sets of initial conditions. Both solutions initially trace the unstable branch $4_{u}^{-}$and both move away along the unstable manifold. In one case the solution is re-captured by the stable branch $4_{s}^{-}$. For the other, 
however, even though the distance from $4_{u}^{-}$is not appreciably greater, the phase point is not recaptured by $4_{s}^{-}$.

\section{Mode doubling transitions under perturbed kinetics}

The mode-doubling failure at low growth rates that we observe appears to be due to accumulation of small errors. This motivates us to consider the influence of small perturbations to the Schnakenberg kinetics. We note that an application of this theory of pattern formation to a biological setting requires us to move away from the idealisation that Eqs. (1)-(3) present. Mode-doubling failure may be presented as an erroneous solution of (4)-(6), but it is a valid phenomenon away from the idealised state or in a noisy environment.

There are many possible ways by which perturbations to the Schnakenberg kinetics may be introduced. Such perturbations could account for spatio-temporal variation in the rate parameters $k_{i}$ or in the source concentrations $[A]$ and $[B]$. For simplicity, we consider perturbations to the source concentrations which are likely to be much larger than those which arise from a variation in the kinetic rate parameters.

The effect of this variation is to replace the terms $a$ and $b$ in Eqs. (4), (5), (8) and (9) by appropriate spatio-temporally varying terms. Under suitable assumptions about the spatial structure of the perturbations we restrict our attention to the perturbations to the system (8)-(12).

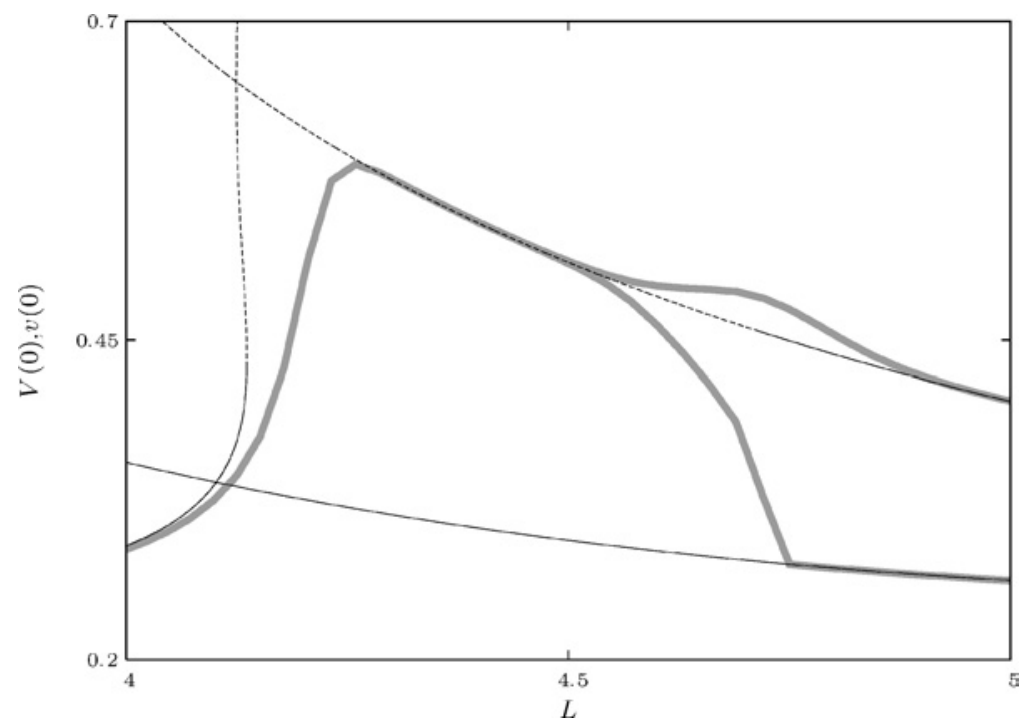

Fig. 7 Unreliable mode doubling for $\rho=0.000136$ across the tracing of $4-$. Two initial states close to the homogeneous steady state, such as used for Fig. 3, are chosen: for one state mode doubling fails; for the other mode doubling succeeds by virtue of recapture by $4_{s}^{-}$. 
The first class of perturbations that we study is that of temporally constant perturbations. In (4), then, we replace $a$ by $a+\varepsilon_{a} \sum_{i=0}^{N-1} a_{i} \cos (\pi i x)$ and similarly for $b$ in (5). These changes propagate to (8)-(11) and we introduce the convention that the perturbations are normalised by $\sum a_{i}^{2}+b_{i}^{2}=1$ and set $\varepsilon_{a}=\varepsilon_{b}$.

During growth we require for mode doubling to fail that the source of $U$ or $V$ be spatially asymmetric. A simple case, therefore, is to consider the normalised perturbation

$$
a_{i}=\left\{\begin{array}{ll}
\frac{10 \sqrt{101}}{101} & i=1 \\
0 & \text { otherwise }
\end{array} ; \quad b_{i}= \begin{cases}\frac{-\sqrt{101}}{101} & i=1 \\
0 & \text { otherwise }\end{cases}\right.
$$

which has the ratio of $U$ and $V$ components of the perturbation of the same magnitude as the ratio of the steady state pattern components.

Under these perturbed kinetics with $\varepsilon=1 \times 10^{-5}$ we see continued applicability of the quasi-steady state assumption and tracing of the steady state branches. As for the solutions of the Schnakenberg PDE system with the spatial discretisation method we see the possible occurrence of mode-doubling failure. This modedoubling failure (for $\rho<0.0002$ ) once again follows from movement away from the unstable $4_{u}^{-}$towards $3_{s}^{ \pm}$. Unlike for the previous case, however, the critical growth rate window arises purely from the influence of the initial conditions, and the window is very narrow.

We find the critical growth rate window becomes wider for the perturbed ODE form with the introduction of temporally varying perturbations. These perturbations can take many forms. Motivated by biological systems we consider the cases where $a_{i}$ and $b_{i}$ vary.

Spatially correlated perturbations similar to coloured noise can be implemented by sampling $a_{i}$ and $b_{i}$ over time from the distributions

$$
a_{i}, b_{i} \in\left[-\frac{\tilde{\varepsilon}}{(i+1)}, \frac{\tilde{\varepsilon}}{(i+1)}\right],
$$

with

$$
\tilde{\varepsilon}=\varepsilon\left(\sum_{i=0}^{N-1} \frac{1}{i+1}\right)^{-1}
$$

With this basic form of spatially correlated perturbation we can determine the form of the corresponding critical growth rate windows for each mode-doubling transition. In Fig. 8 we present such windows for the transitions $2^{-} \rightarrow 4^{-}, 3^{-} \rightarrow 6^{-}$ and $4^{-} \rightarrow 8^{-}$. 


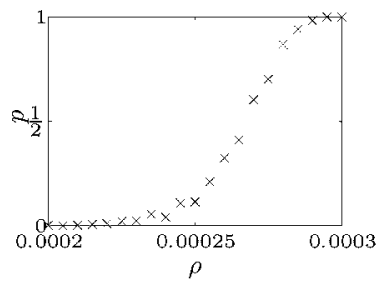

(a) $2^{-} \rightarrow 4^{-}$

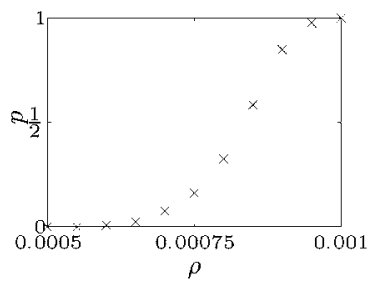

(b) $3^{-} \rightarrow 6^{-}$

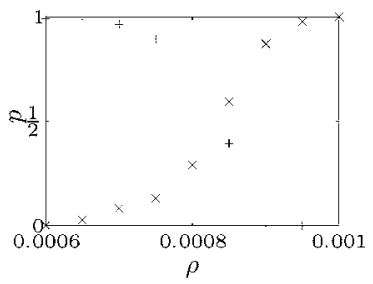

(c) $4^{-} \rightarrow 8^{-}$

Fig. 8 Critical growth rate windows for the transitions $2^{-} \rightarrow 4^{-}, 3^{-} \rightarrow 6^{-}$and $4^{-} \rightarrow 8^{-}$of (8)-(12) subject to the kinetic perturbation (14). In each, $\times$ denotes the transition mode-doubling probability, whilst in (c), + represents the probability that mode-doubling failure is by $4^{-} \rightarrow 5^{ \pm}$.

\section{Domain growth and the robustness problem}

The critical growth rate windows of Fig. 8 show that, for a consistent perturbation characteristic, the growth rate at which mode doubling can be viewed as reliable varies with the transition. This raises the possibility of robust pattern selection through a pattern sequence involving a failure of mode doubling. Whereas persistent mode doubling provides for robust selection of pattern modes of the form $\left(2^{n}\right)^{-}$for the appropriate final domain length it is not possible to robustly generate further pattern modes when the pattern $1^{ \pm}$is initially expressed.

When mode-doubling failure is included, however, the pattern $6_{s}^{-}$is selectable for a range of final domain lengths. A sequence such as $1^{ \pm} \rightarrow 2^{-} \rightarrow 3^{ \pm} \rightarrow 6^{-}$, should it arise, is precisely this selection. The possibility of robust selection of $6_{s}^{-}$ arises with (loose) control of the growth rate of the domain, as indicated by the critical growth rate windows for the final two transitions.

We also note that control of the perturbations may further aid both the selection of polarity of branch following mode-doubling failure and increase the range of growth rates suitable for robust selection. A constant pre-pattern, for example, in addition to, but of a greater magnitude than, stochastic perturbations may be such a guide.

\section{Conclusions}

In this paper we have examined mode transition in a reaction-diffusion system solved on an exponentially growing domain. We have shown that the typical long term mode-doubling behaviour breaks down due to noise induced by our numerical scheme. Although, this is an artifact of our solution technique, it is not biologically irrelevant, because processes in biology have to operate under constant exposure to noise. This motivates us to consider the case of noise in parameter values and to show that this also contributes to mode-doubling failure.

Applications of reaction-diffusion theory to biological pattern formation have been studied in great detail. While its relevance to biology is still controversial, it has been shown that Turing structures do arise in chemistry (DeKepper et al., 1991; Ouyang and Swinney, 1991). It is still unclear as to whether morphogen patterns in biology do arise via diffusion-driven instability and whether such a mech- 
anism presents stable patterns. Moreover, it is surprising that little attention has been paid to the effects of domain growth, given that growth is one of the main characteristics of development. It was shown by Kondo and Asai (1995) that pigmentation patterns on certain Angelfish followed a transition sequence consistent with that predicted by a Turing type model. This motivated the work of Crampin et al. (1999), which showed that domain growth could be used to select robustly certain types of patterns.

In this paper, we have examined why mode-doubling on an exponentially growing one-dimensional domain breaks down under very slow growth rate, specifically when symmetry arguments would suggest otherwise. We have shown that this phenomenon appears to arise due to noise. At certain transition points, instability of the solution arises due to a saddle point and the solution must trace an unstable branch for some time before it reaches a stable branch. The presence of noise can throw the solution off this branch and we propose this as a mechanism for modedoubling failure.

The results in this paper are for a single point in parameter space for the Schnakenberg model. In current work we are examining this in more detail with application to other models, but we note that the behaviour presented here is widespread. In particular, for the Gray-Scott system, which has been widely studied (Kolokolnikov et al., 2005) in many of its behavioural regimes, we see mode doubling with the possibility of failure when the pattern sequence is driven by domain growth.

\section{Acknowledgements}

IB acknowledges the EPSRC for a graduate studentship. EJC thanks the Wellcome Trust and the New Zealand Institute for Mathematics and its Applications. The results of this work were presented by PKM in Banff in 2003 at the meeting to honour the legacy of Lee Segel.

\section{References}

Bard, J., Lauder, I., 1974. How well does Turing's theory of morphogenesis work? J. Theor. Biol. $45,501-531$

Crampin, E.J., 2000. Reaction-diffusion patterns on growing domains. DPhil thesis, University of Oxford, UK.

Crampin, E.J., Gaffney, E.A., Maini, P.K., 1999. Reaction and diffusion on growing domains: Scenarios for robust pattern formation. Bull. Math. Biol. 61(6), 1093-1120.

Crampin, E.J., Gaffney, E.A., Maini, P.K., 2002. Mode doubling and tripling in reaction-diffusion patterns on growing domains: A piecewise linear model. J. Math. Biol. 44(2), 107-128.

DeKepper, D., Castets, V., Dulos, E., Boissonade, J., 1991. Turing-type chemical patterns in the chlorite-iodide-malonic acid reaction. Physica D 61, 161-169.

Dillon, R., Maini, P.K., Othmer, H.G., 1994. Pattern formation in generalised Turing systems I: Steady state patterns in systems with mixed boundary conditions. J. Math. Biol. 32, 345-393.

Doedel, E.J., Paffenroth, R.C., Champneys, A.R., Fairgrieve, T.F., Kuznetsov, Y.A., Sandstede, B., Wang X., 1997. AUTO 2000: Continuation and Bifurcation Software for Ordinary Differential Equations (with HomCont). Technical Report, Caltech, February 2001.

Hayes, C., Brown, J.M., Lyon, M.F., Morriss-Kay, G.M., 1998. Sonic hedgehog is not required for polarising activity in the Doublefoot mutant mouse limb bud. Development 125, 351-357. 
Iron, D., Wei, J., Winter, M., 2004. Stability analysis of Turing patterns generated by the Schnakenberg Model. J. Math. Biol. 49(4), 358-390

Keller, E.F., Segel, L.A., 1970. The initiation of slime mold aggregation viewed as an instability. J. theor. Biol. 26, 399-415.

Kolokolnikov, T., Ward, M.J., Wei. J., 2005. The existence and stability of spike equilibria in the one-dimensional Gray-Scott model on a finite domain. Appl. Math. Lett. 18(8), 951-956.

Kondo, S., Asai, R., 1995. A reaction-diffusion wave on the skin of the marine angelfish Pomacanthus. Nature 376, 765-768

Meinhardt, H., 1995. The Algorithmic Beauty of Sea Shells. Springer, Heidelberg.

Murray, J.D., 2003. Mathematical Biology. 3rd edition in 2 volumes: Mathematical Biology: II. Spatial Models and Biomedical Applications, Springer, New York.

Ouyang, Q., Swinney, H.L., 1991. Transition from a uniform state to hexagonal and striped Turing patterns. Nature 353(6336), 610-612.

Segel, L.A., Jackson, J.L., 1972. Dissipative structure: An explanation and an ecological example. J. Theor. Biol. 37, 545-559.

Turing, A.M., 1952. The chemical basis of morphogenesis. Phil. Trans. R. Soc. Lond. B 237, 37-72.

Yoon, H.-S., Golden, J.W., 1998. Heterocyst pattern formation controlled by a diffusible peptide. Science 282, 935-938. 